\title{
Reading and writing operations of memory device in micro-electromechanical resonator
}

\author{
Atsushi Yao $^{1 \mathrm{a})}$ and Takashi Hikihara ${ }^{1 \mathrm{~b})}$ \\ ${ }^{1}$ Department of Electrical Engineering, Kyoto University \\ Katsura, Nishikyo, Kyoto, 615-8510 Japan \\ a)yao@dove.kuee.kyoto-u.ac.jp, \\ b) hikihara.takashi.2n@kyoto-u.ac.jp
}

\begin{abstract}
Micro-electromechanical resonators substantially exhibit bistable and hysteretic response when nonlinear characteristics appear. Badzey et al. reported that the nonlinear micro-electromechanical resonators can be used as a mechanical 1 bit memory. Based on their results, the authors propose reading and writing operations of the memory device. The reading and writing operations imply a displacement measurement and a switching of two stable periodic vibrations, respectively. In this paper, we realize a displacement measurement along an approach avoiding supplemented sensors. In addition, we achieve the switching operation between two coexisting periodic states by a displacement feedback control.
\end{abstract}

Keywords: MEMS resonator, memory device, reading operation, writing operation, nonlinear

Classification: Micro- or nano-electromechanical systems

\section{References}

[1] V. Kaajakari, Practical MEMS, Small Gear Publishing, Las Vegas, 2009.

[2] J. A. Pelesko and D. H. Bernstein, Modeling MEMS and NEMS, CHAMPMAN \& HALL/CRC, Boca Raton, 2003.

[3] A. Frangi, C. Cercignani, S. Mukherjee, and N. Aluru, Advances in Multiphysics Simulation and Experimental Testing of MEMS, Imperial College Press, London, 2008.

[4] R. L. Badzey, G. Zolfagharkhani, A. Gaidarzhy, and P. Mohanty, "A controllable nanomechanical memory element," Applied Physics Letters, vol. 85, no. 16, pp. 3587-3589, 2004.

[5] I. Mahboob and H. Yamaguchi, "Bit storage and bit flip operations in an electromechanical oscillator," Nature Nanotechnology, vol. 3, no. 5, pp. 275-279, 2008.

[6] H. Noh, S. Shim, M. Jung, Z. G. Khim, and J. Kim, "A mechanical memory with a dc modulation of nonlinear resonance," Applied Physics Letters, vol. 97, no. 3, pp. 033116-1-033116-3, 2010. 
[7] Q. P. Unterreithmeier, T. Faust, and J. P. Kotthaus, "Nonlinear switching dynamics in a nanomechanical resonator," Physical Review B, vol. 81, no. 24, pp. 241405-1-241405-4, 2010.

[8] Q. P. Unterreithmeier, E. M. Weig, and J. P. Kotthaus, "Universal transduction scheme for nanomechanical systems based on dielectric forces," Nature, vol. 458, no. 7241, pp. 1001-1004, 2009.

[9] R. M. C. Mestrom, R. H. B. Fey, J. T. M. van Beek, K. L. Phan, and H. Nijmeijer, "Modelling the dynamics of a MEMS resonator: Simulations and experiments," Sensors and Actuators A: Physical, vol. 142, no. 1, pp. 306-315, 2008.

[10] I. A. Ivan, M. Rakotondrabe, P. Lutz, and N. Chaillet, "Quasistatic displacement self-sensing method for cantilevered piezoelectric actuators," Review of Scientific Instruments, vol. 80, no. 6, pp. 065102-1-065102-8, 2009.

[11] K. Matsuda, Y. Okada, and J. Tani, "Self-sensing magnetic bearing using the differential transformer principle," Transactions of the Japan Society of Mechanical Engineers. C, vol. 63, no. 609, pp. 1441-1447, 1997 (in Japanese).

[12] S. Naik and T. Hikihara, "Characterization of a MEMS resonator with extended hysteresis," IEICE Electron. Express, vol. 8, no. 5, pp. 291-298, 2011.

[13] S. Naik, Investigation of synchronization in a ring of coupled MEMS resonators, Doctoral Dissertation (Kyoto University), 2011.

[14] V. Kempe, Inertial MEMS: Principles and Practice, Cambridge University Press, Cambridge, 2011.

\section{Introduction}

Micro-electromechanical resonators, which are fabricated in micro-electromechanical systems (MEMS) technology, have been developed as sensor elements, filters, and frequency references [1]. MEMS resonators are known to produce nonlinear responses at large amplitude excitation [1]. Due to hard or soft spring effect [2], an amplitude-frequency response curve around resonance tends to bend toward higher or lower frequencies at the nonlinear responses [1,3]. MEMS resonators exhibit hysteresis characteristics for upsweep and downsweep of the frequency because of nonlinear restoring force $[1,3]$. In other words, nonlinear MEMS resonators have two stable vibrating states at a single frequency in the hysteresis $[1,3]$. Badzey et al. reported that these nonlinear MEMS resonators can be applied to a memory device [4]. Based on their results, we try to demonstrate reading and writing operations of the memory device. For the memory device in micro- and nanoelectromechanical resonators $[4,5,6,7]$, the reading and writing operations imply a displacement measurement and a switching of two stable periodic vibrations.

Recently, Unterreithmeier et al. suggested a switching between two coexisting stable states in a nonlinear MEMS resonator at a constant excitation frequency [7]. They applied a radio-frequency pulse to control two stable states. They experimentally showed clear possibilities of switching states. 
Their results motivated us to find other appropriate continuous control methods for the practical use of MEMS resonators based on feedback control. In this paper, the switching is experimentally performed between two coexisting stable states by a displacement feedback control as a writing operation of the memory device in a MEMS resonator.

For the displacement feedback control, the displacement measurement is required. The displacement measurements of MEMS resonators, based on piezoelectric element [5], photodetector [8], electric circuit [9], and so on, have been recently studied. We propose the displacement measurement by using a self-sensing method for the combined structure of actuator and sensor in a MEMS resonator. That is, the MEMS resonator has a comb-drive as a forcing actuator, which is simultaneously used as a displacement sensor. The self-sensing can be a solution for the integration and the simplification of whole system [10, 11]. From this standpoint, we perform a self-sensing method, based on the measurement of the current through the capacitor of the MEMS resonator. As mentioned above, this displacement measurement also represents a reading operation of the memory device in a MEMS resonator.

\section{Fabricated MEMS resonator and its capacitance}

As the suitable MEMS resonator, a comb-drive resonator is designed and fabricated to obtain hysteretic characteristics during upsweep and downsweep of frequency as shown in Fig. $1[12,13]$. Figure 1 (a) illustrates a perforated mass suspended by springs, which are connected to anchor [12]. As the anchor is connected to ground and either of the right or the left electrode to ac voltage source, the mass vibrates only in $x$-direction. Figure 1 (b) depicts a cross section of the dotted lines of Fig. 1 (a). The substrate and oxide layers underneath the movable structure are removed as shown in Fig. 1 (b).

When the mass vibrates, the capacitance is given by

$$
C_{1}=2 \varepsilon N \frac{h(l+x)}{d}=2 \varepsilon N \frac{h l}{d}+2 \varepsilon N \frac{h x}{d}=C_{0}+C(x),
$$
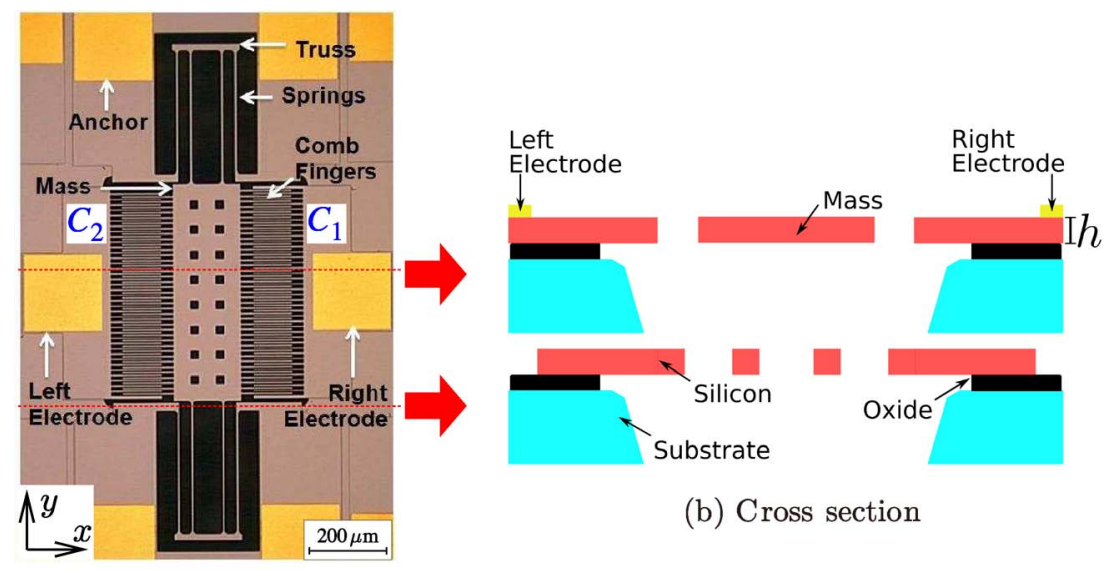

(b) Cross section

(a) Top view 


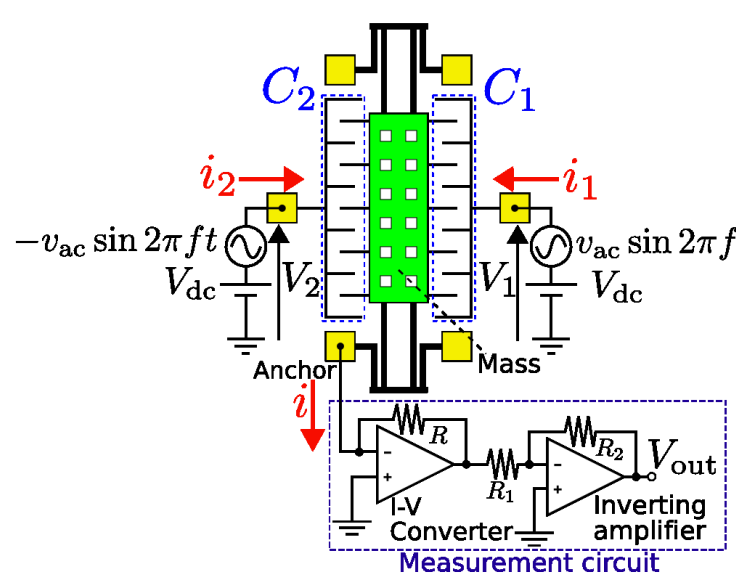

(a) Schematic diagram of differential measurement

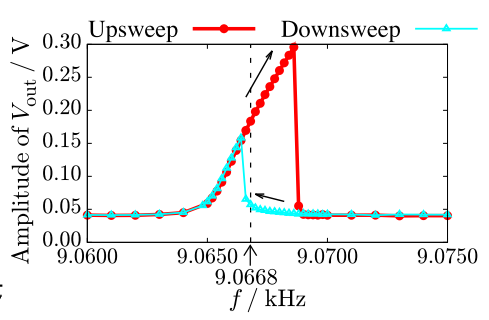

(b) Frequency response curve

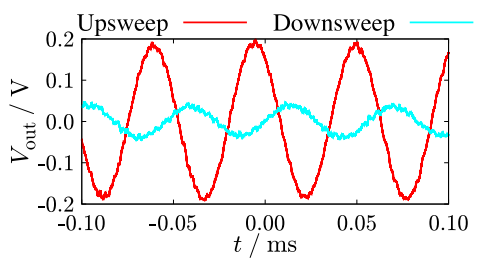

(c) Two stable periodic vibrations at $9.0668 \mathrm{kHz}$

Fig. 2. Displacement measurement by differential configuration

$$
C_{2}=2 \varepsilon N \frac{h(l-x)}{d}=2 \varepsilon N \frac{h l}{d}-2 \varepsilon N \frac{h x}{d}=C_{0}-C(x),
$$

where $C_{1}$ denotes the capacitance of the right electrode, $C_{2}$ the capacitance of the left electrode, $x$ the displacement, $d(=3 \mu \mathrm{m})$ the gap between the fingers, $l(=100 \mu \mathrm{m})$ the initial overlap between the fingers, $\varepsilon(=8.85 \times$ $\left.10^{-12} \mathrm{~F} / \mathrm{m}\right)$ the permittivity, $N(=39)$ the comb number, $h(=25 \mu \mathrm{m})$ the finger height, $C_{0}\left(=5.8 \times 10^{-13} \mathrm{~F}\right)$ the initial capacitance, and $C(x)$ the change of capacitance. When the displacement $x$ is set at $5 \mu \mathrm{m}, C(x)$ becomes $2.9 \times 10^{-14} \mathrm{~F}$. A parasitic capacitance between bond pad and substrate is estimated as $1.4 \times 10^{-12} \mathrm{~F}$.

\section{Displacement measurement}

In this section, we explain a displacement measurement of comb-drive resonator by using the differential measurement [14] as shown in Fig. 2 (a). That is, the voltage of right electrode was excited by $V_{\mathrm{dc}}+v_{\mathrm{ac}} \sin 2 \pi f t$ and the left by $V_{\mathrm{dc}}-v_{\mathrm{ac}} \sin 2 \pi f t$, respectively.

When the mass vibrates only in $x$-direction, a current of the anchor flows through the capacitor depending on the displacement of a comb-drive resonator [1]. However, the current is based not only on the displacement but also on the others when either of the electrodes is connected to the voltage source and the other to ground [1]. Therefore, the displacement can be extracted by the differential measurement of current (see Fig. 2 (a)).

In the measurement, the excitation force $F$ and the sum of the current through the right and left capacitor $i$ are obtained by the following equations:

$$
\begin{aligned}
F & =\left(\frac{\partial C_{1}}{\partial x}\right) \frac{V_{1}^{2}}{2}+\left(\frac{\partial C_{2}}{\partial x}\right) \frac{V_{2}^{2}}{2}=4 \varepsilon N \frac{h}{d} V_{\mathrm{dc}} v_{\mathrm{ac}} \sin 2 \pi f t, \\
i & =i_{1}+i_{2}=\frac{\partial\left(C_{1} V_{1}\right)}{\partial t}+\frac{\partial\left(C_{2} V_{2}\right)}{\partial t} \\
& =\left(8 \pi f \varepsilon N \frac{h}{d} v_{\mathrm{ac}} \cos 2 \pi f t\right) x+\left(4 \varepsilon N \frac{h}{d} v_{\mathrm{ac}} \sin 2 \pi f t\right) \frac{\mathrm{d} x}{\mathrm{~d} t},
\end{aligned}
$$


where $V_{1}\left(=V_{\mathrm{dc}}+v_{\mathrm{ac}} \sin 2 \pi f t\right)$ denotes the applied voltage of the right electrode, $V_{2}\left(=V_{\mathrm{dc}}-v_{\mathrm{ac}} \sin 2 \pi f t\right)$ the applied voltage of the left electrode, $i_{1}$ the current through the right capacitor $C_{1}, i_{2}$ the current through the left capacitor $C_{2}$, and $f$ the excitation frequency. In the experiments of this paper, the dc bias voltage $V_{\mathrm{dc}}$ and the ac excitation amplitude $v_{\mathrm{ac}}$ are set at $-0.15 \mathrm{~V}$ and $0.7 \mathrm{~V}$ in vacuum at $10 \mathrm{~Pa}$.

If the displacement $x(t)$ is assumed as $A_{0} \sin (2 \pi f t+\phi)$, the current $i$ is obtained as

$$
i=8 \pi f \varepsilon N \frac{h}{d} v_{\mathrm{ac}} A_{0} \sin (4 \pi f t+\phi),
$$

where $A_{0}$ and $\phi$ denote the amplitude and the phase of the displacement.

The current $i$ is converted to the output voltage $V_{\text {out }}$ by two operational amplifiers as in Fig. 2 (a). The first stage operational amplifier works as the current-to-voltage (I-V) converter and the second as the inverting amplifier. The output voltage $V_{\text {out }}$ is obtained as

$$
V_{\text {out }}=R \frac{R_{2}}{R_{1}} i=8 \pi f R \frac{R_{2}}{R_{1}} \varepsilon N \frac{h}{d} v_{\text {ac }} A_{0} \sin (4 \pi f t+\phi) .
$$

The resistors $R, R_{1}$, and $R_{2}$ are set at $1 \mathrm{M} \Omega, 1 \mathrm{k} \Omega$, and $100 \mathrm{k} \Omega$, respectively. We can confirm that $V_{\text {out }}$ depends on the amplitude $A_{0}$ and the phase $\phi$ of the displacement $x$. The frequency of the output voltage becomes twice the excitation frequency $f$.

Figure 2 (b) shows the experimentally obtained frequency response curve of $V_{\text {out }}$. In Fig. 2(b), red and aqua lines show the response at upsweep and downsweep of frequency, respectively. We confirmed that the MEMS resonator has hysteresis characteristics to frequency and two stable states coexist at the hysteresis region.

Figure 2 (c) depicts the oscillogram of two stable periodic vibrations at $9.0668 \mathrm{kHz}$. The red and aqua lines are obtained by the average of 16 times measurements. It was found that these two stable periodic vibrations are obviously distinguished by the amplitude and the phase. In the following experiments, the excitation frequency is fixed at $9.0668 \mathrm{kHz}$.

\section{Switching between two stable periodic vibrations}

In this section, the switching operation between two coexisting states is discussed by a displacement feedback control at a constant excitation frequency. It has already been explained that the output voltage $V_{\text {out }}$ is returned as a proportional signal to the displacement by using the differential measurement in Sec. 3. Therefore, based on the output voltage, we consider the switching by the feedback control as shown in Fig. 3 (a).

Here, we need to apply the control input to the MEMS resonator without any influence to measurement. Note that the excitation force $F$ depends on both the ac excitation amplitude $v_{\mathrm{ac}}$ and the dc bias voltage $V_{\mathrm{dc}}$ but the output voltage $V_{\text {out }}$ only on the ac excitation amplitude $v_{\text {ac }}$ as given in Eqs. (3) and (6). Based on the feature, the control input is decided to apply as a slowly changed dc voltage to MEMS resonator. 


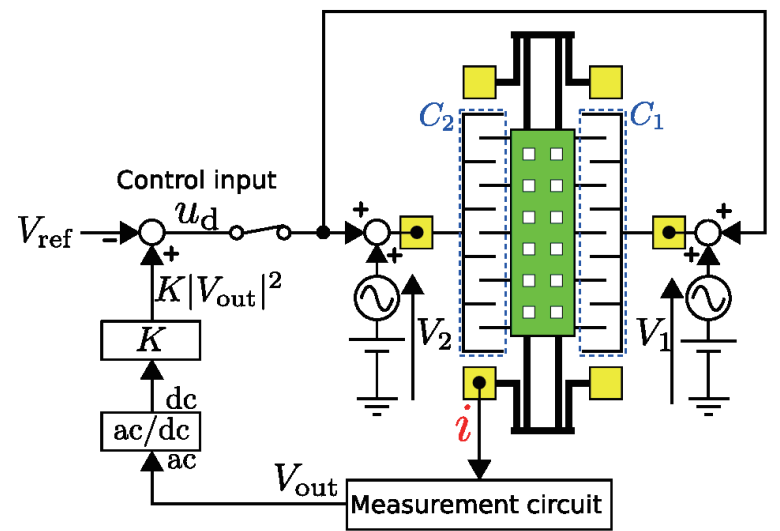

(a) Control system for switching of two vibrations by feedback control in MEMS resonator

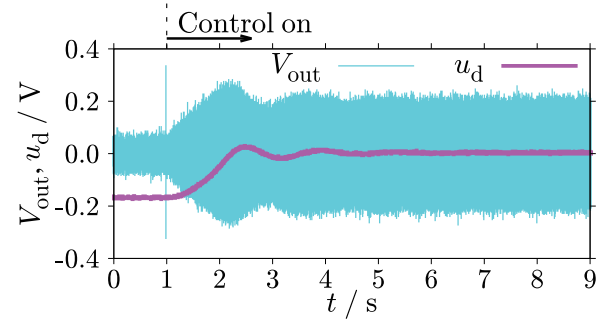

(b) Switching from small to large amplitude vibration

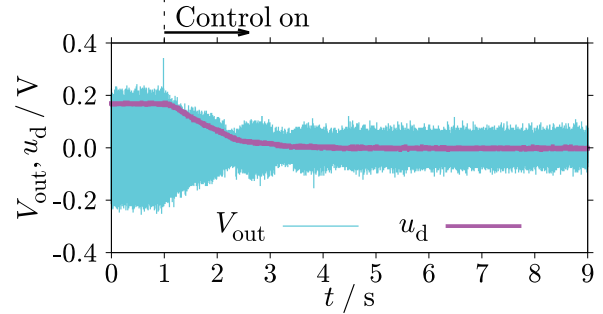

(c) Switching from large to small amplitude vibration

Fig. 3. Switching between two stable periodic vibrations

Figure 3 (a) shows the control system for the switching of two vibrations by the proposed displacement feedback control to the MEMS resonator. The output ac voltage $V_{\text {out }}$ is converted to the dc voltage $\left|V_{\text {out }}\right|^{2}$ by an analog multiplier and a low pass filter of the operational amplifier. The control input $u_{\mathrm{d}}$ is given as a slowly changed dc voltage by

$$
u_{\mathrm{d}}=-V_{\text {ref }}+K\left|V_{\text {out }}\right|^{2} \text {, }
$$

where $K(=5.1)$ is the feedback gain and $V_{\text {ref }}$ the external reference signal. The external reference signal is set at $K\left|V_{\text {out }}^{\mathrm{L}}\right|^{2}$ when the state is requested to switch to the large amplitude vibration. When the state is switched to the small amplitude vibration, the external reference signal is reset at $K\left|V_{\text {out }}^{\mathrm{S}}\right|^{2}$. Here, $V_{\text {out }}^{L}$ and $V_{\text {out }}^{S}$ show the targeted output voltage for the large and small amplitude vibrations. As a result, the excitation force $F$ is given with control input $u_{\mathrm{d}}$ by

$$
F=4 \varepsilon N \frac{h}{d}\left(V_{\mathrm{dc}}+u_{\mathrm{d}}\right) v_{\mathrm{ac}} \sin 2 \pi f t .
$$

Figures $3(\mathrm{~b})$ and 3 (c) show the vibrations switched by the control input $u_{\mathrm{d}}$. In these figures, aqua and purple lines correspond to the output voltage $V_{\text {out }}$ and the control input $u_{\mathrm{d}}$, respectively. The control input was applied at $1 \mathrm{~s}$ from the beginning of the oscillogram. Figure $3(\mathrm{~b})$ shows the result of the switching from small to large amplitude vibrations. It was found that the transition is slow from small to large amplitude vibrations. It took around 
$5 \mathrm{~s}$ until the conversion. After the switching was completed, the control input $u_{\mathrm{d}}$ almost became null. Figure 3 (c) shows the result of the switching from large to small amplitude vibrations. It was observed that the converged state remains with a small amplitude periodic vibration in the comb-drive resonator. The switching was completed after $4 \mathrm{~s}$ and the control input $u_{\mathrm{d}}$ almost disappeared. At the onset of the control $(1 \mathrm{~s})$, the output voltage $V_{\text {out }}$ showed a surge each in Figs. 3 (b) and 3 (c). However, there did not happen any fault of switching operation.

\section{Conclusion}

For the achievement of reading and writing operations of a memory device in a nonlinear MEMS resonator, we investigated measurement and switching of two distinct vibrations. It was shown that the sum of the current through the capacitor only depends on the displacement in the differential measurement. We confirmed, without additional sensors except the current measurement, that two vibrations can be distinguished in the amplitude and the phase. Therefore, the reading operation can be implemented by the selfsensing method. It was also cleared that the state information is kept through the measurement.

Second, based on the displacement measurement, we achieved the switching between two stable periodic vibrations. The dc voltage bias was added to the excitation voltage for avoiding the influence to measurement. As a result, the switching operation was achieved between two coexisting stable states by the displacement feedback control at a constant excitation frequency in the hysteresis.

This paper experimentally realized the reading and the writing operations of the memory device in the MEMS resonator by the self-sensing method and the displacement feedback control. We believe that these operations will be keys to develop new functions in the application of MEMS.

\section{Acknowledgments}

We would like to show our appreciation to Dr. S. Naik, SPAWAR, USA, for the discussion about the design of MEMS resonator. This research was partially supported by Global COE of Kyoto University, Regional Innovation Cluster Program "Kyoto Environmental Nanotechnology Cluster," and JSPS KAKENHI (Grant-in-Aid for Exploratory Research) $\sharp 21656074$. 